\title{
Dinitrobenzene sensing utilizing chitosan-based thin films optical fluorescence sensors via linear and nonlinear excitation
}

\begin{abstract}
We presented here the findings of linear and nonlinear excitation of an optical fluorescence sensor enhanced with chitosan-based thin films coated on tapered optical fiber for detecting of dinitrobenzene solution compound. The absorbance peak of linear excitation at $441.2 \mathrm{~nm}$ shows a small shift to from $540.8 \mathrm{~nm}$ to $541.3 \mathrm{~nm}$ when $0.003 \mathrm{~g} / \mathrm{ml}$ dinitrobenzene was diluted in acetone. Time resolved fluorescence resulting in decreasing of fluorescence intensities was recorded for both linear and nonlinear excitation. Both showed an agreement of quenching of fluorescence up to $30 \%$ of its initial fluorescence due to the free electron transfer from chitosan thin film to the dinitrobenzene. Nonlinear excitation gives more rapid time-resolved florescence after 4 minutes compared to 6 minutes in linear excitation.
\end{abstract}

Keyword: Chitosan; Dinitrobenzene; Linear excitation; Nonlinear excitation; Optical timeresolved fluorescence sensor 